\title{
Maintaining and Testing Separability in Demand Systems
}

\author{
Giancarlo Moschini; Daniele Moro; Richard D. Green
}

American Journal of Agricultural Economics, Vol. 76, No. 1. (Feb., 1994), pp. 61-73.

Stable URL:

http://links.jstor.org/sici?sici=0002-9092\%28199402\%2976\%3A1\%3C61\%3AMATSID\%3E2.0.CO\%3B2-K

American Journal of Agricultural Economics is currently published by American Agricultural Economics Association.

Your use of the JSTOR archive indicates your acceptance of JSTOR's Terms and Conditions of Use, available at

http://www.jstor.org/about/terms.html. JSTOR's Terms and Conditions of Use provides, in part, that unless you have obtained prior permission, you may not download an entire issue of a journal or multiple copies of articles, and you may use content in the JSTOR archive only for your personal, non-commercial use.

Please contact the publisher regarding any further use of this work. Publisher contact information may be obtained at http://www.jstor.org/journals/aaea.html.

Each copy of any part of a JSTOR transmission must contain the same copyright notice that appears on the screen or printed page of such transmission.

The JSTOR Archive is a trusted digital repository providing for long-term preservation and access to leading academic journals and scholarly literature from around the world. The Archive is supported by libraries, scholarly societies, publishers, and foundations. It is an initiative of JSTOR, a not-for-profit organization with a mission to help the scholarly community take advantage of advances in technology. For more information regarding JSTOR, please contact support@ jstor.org. 


\title{
Maintaining and Testing Separability in Demand Systems
}

\author{
Giancarlo Moschini, Daniele Moro, and Richard D. Green
}

\begin{abstract}
We derive a general elasticity representation of the necessary and sufficient conditions for direct weak separability of the utility function. Parametric restrictions required to implement the separability conditions are presented for three common demand systems: the Almost Ideal, Translog, and Rotterdam. Our empirical application uses the Rotterdam model to test a few separable structures within a complete U.S. demand system emphasizing food commodities. Results, based on size-corrected likelihood ratio tests, provide support for commonly used separability assumptions about food and meat demand.
\end{abstract}

Key words: Almost Ideal Demand System, conditional demand systems, demand analysis, duality, likelihood ratio test, Rotterdam model, separability, Translog model.

The concept of separability, originally introduced by Leontief and Sono, can be extremely useful for economic modeling because of its wideranging implications for the existence of consistent aggregates and the decentralization of optimization decisions (Blackorby, Primont, and Russell 1978). In demand analysis, an often invoked separability assumption leads to the specification of conditional (second stage) demand systems. For example, it is common to model demand for meats (beef, pork, and poultry) as a function of the price of these three meat aggregates and of total meat expenditure. Such a procedure is justified if the direct utility function is weakly separable in the appropriate partition, which provides the necessary and sufficient condition for conditional demand functions to exist.

There are at least two undesirable features associated with the empirical use of conditional demand systems. First, the first-stage income allocation often is left unspecified, or is ad hoc, which makes the resulting elasticity estimates of limited value. Second, although direct weak separability guarantees the existence of conditional demand systems, econometric problems

Giancarlo Moschini is an associate professor, Department of Economics, Iowa State University; Daniele Moro is a researcher, Istituto di Economia, Università Cattolica, Piacenza (Italy); and, Richard Green is a professor, Department of Agricultural Economics, University of California-Davis.

We thank Jeff LaFrance and two anonymous reviewers for helpful comments. Remaining errors are our own. This is Journal Paper No. J-15235 of the Iowa Agriculture and Home Economics Experiment Station, Project No. 2875.

Review coordinated by Steven Buccola. still may exist in estimation because group expenditures are endogenous (LaFrance). These limitations could be eschewed if weak separability restrictions were built into a full demand system. Such a procedure, while still resulting in considerable degrees of freedom gain, would be free of the expenditure endogeneity problem discussed by LaFrance. Moreover, it would simultaneously account for the first stage income allocation in a theoretically consistent fashion, and yield unconditional demand elasticities suitable for policy and welfare analysis. However, the alternative of maintaining separability restrictions within a full demand system is seldom adopted in empirical analysis. The first objective of this paper is to show a systematic procedure for maintaining separability restrictions in a full demand system within commonly used demand models.

Although more attention could be paid to maintaining separability in demand models, the analyst cannot escape the fact that the convenience of an assumption is no substitute for its truth. Hence, legitimate interest exists in the empirical test of the validity of separability assumptions in demand models. A few demand studies have undertaken to test types of separability assumptions, including Byron, Jorgenson and Lau; Barnett (1979b); and Barnett and Choi. Pudney addresses the somewhat different issue of the determination of the appropriate grouping pattern, and related work is found in Baccouche and Laisney; and Nicol. Hayes, Wahl, and Williams aimed at testing quasi-separabil- 
ity, but such separability is consistent with direct weak separability only if the subutility groups are homothetic (thus, in general, it cannot be used to justify second-stage demand systems). Alston, Carter, Green, and Pick followed Winters and tested necessary (but not sufficient) conditions for direct weak separability. Eales and Unnevehr apply separability arguments to investigate alternative meat aggregates, but end up testing only a subset of the required restrictions. Such overlooked details suggest that further attention to testing separability may be worthwhile. Our setup provides a unified framework for specifying and testing separability. It allows us to illustrate and clarify separability restrictions for alternative demand systems (such as the Almost Ideal, Translog, and Rotterdam), which are typically derived in a very different fashion.

\section{Direct Separability and Demand Analysis}

To characterize separability, it is important to distinguish between symmetric and asymmetric separable structures (Blackorby, Primont, and Russell 1978). To review briefly, let $\mathbf{q}$ $=\left(q_{1}, \ldots, q_{n}\right)$ denote the vector of consumer goods, $\mathbf{p}=\left(p_{1}, \ldots, p_{n}\right)$ denote the corresponding nominal price vector, and $y$ denote total expenditures on the $n$ goods (income, for short). The set of indices of the $n$ goods is $I=\{1, \ldots$, $n\}$, and these goods can be ordered in $S$ separable groups defined by the mutually exclusive and exhaustive partition $\hat{I}=\left\{I_{1}, \ldots, I_{S}\right\}$ of the set $I$. If $U(\mathbf{q})$ is the utility function, then $U(\mathbf{q})$ is symmetrically directly separable in the partition $\hat{I}$ if it can be written as:

$$
U(\mathbf{q})=U^{0}\left[U^{1}\left(\mathbf{q}^{1}\right), U^{2}\left(\mathbf{q}^{2}\right), \ldots, U^{S}\left(\mathbf{q}^{S}\right)\right]
$$

where $U^{s}(\cdot)$ are subutility functions that depend on a subset $\mathbf{q}^{s}$ of goods whose indices are in $I_{S}(s=1, \ldots, S)$. We assume $U^{0}(\cdot)$ and the subutility functions $U^{s}(\cdot)$ satisfy conditions typically required of a utility function (in particular, strong monotonicity, strict quasi-concavity, and differentiability).

It is known that the separable structure in (1) imposes a number of restrictions on the substitution possibilities between goods in different groups. If $h_{i}(\mathbf{p}, u)$ denotes the $i$ th Hicksian (compensated) demand function, where $u$ is a reference utility level, $V(\mathbf{p}, y)$ is the indirect utility function dual to $U(\mathbf{q})$, and $q_{i}(\mathbf{p}, y)$ is the $i$ th Marshallian (ordinary) demand function, Goldman and Uzawa showed that the Slutsky sub- stitution terms between two goods in different groups are proportional to the income effects of the two goods involved:

$$
\frac{\partial h_{i}(\mathbf{p}, V(\mathbf{p}, y))}{\partial p_{k}}=\mu_{g s}(\mathbf{p}, y) \frac{\partial q_{i}(\mathbf{p}, y)}{\partial y} \frac{\partial q_{k}(\mathbf{p}, y)}{\partial y}
$$

for all $i \in I_{g}$ and $k \in I_{s}$, for all $g \neq s$. Note that the proportionality term $\mu_{g s}(\mathbf{p}, y)$ is the same for all goods in the two groups involved. It is important to emphasize that the restrictions in (2) are necessary and sufficient for the weakly separable structure in (1). Hence, (2) summarizes all the relevant restrictions of the separable structure in (1) and can be used to maintain this form of separability or to test it.

Asymmetric separability assumes weaker conditions on the utility function. For a group of goods indexed by $I_{s}$, let $I^{c}$ be the set of indices of all other goods. Then the goods indexed by $I_{s}$ are directly separable from their complement if $U(\mathbf{q})$ can be written as

$$
U(\mathbf{q})=U^{0}\left[\mathbf{q}^{c}, U^{s}\left(\mathbf{q}^{s}\right)\right] .
$$

Blackorby, Davidson, and Schworm point out that Goldman and Uzawa's result does not apply to the asymmetric structure in (3) because their proof essentially required that at least two goods be in any one separable group. Blackorby, Davidson, and Schworm provide alternative versions of the necessary and sufficient conditions for asymmetric weak separability. For our purposes it is useful to present the conditions in a form similar to (2). From their theorem 3 (ii), we have

$$
\begin{aligned}
& \frac{\partial h_{i}(\mathbf{p}, u)}{\partial p_{k}}=\Gamma(\mathbf{p}, u) \frac{\partial \Xi(\mathbf{p}, u)}{\partial p_{i}} \frac{\partial \Xi(\mathbf{p}, u)}{\partial p_{k}} \\
& \frac{\partial h_{i}(\mathbf{p}, u)}{\partial u}=\Gamma(\mathbf{p}, u) \frac{\partial \Xi(\mathbf{p}, u)}{\partial p_{i}} \frac{\partial \Xi(\mathbf{p}, u)}{\partial u}
\end{aligned}
$$

for some appropriately defined functions $\Gamma(\cdot)$ and $\Xi(\cdot)$ and for all $i \in I_{s}$ and $k \in I^{c}$. From the identity $q_{i}(\mathbf{p}, C(\mathbf{p}, u)) \equiv h_{i}(\mathbf{p}, u)$, where $C(\mathbf{p}, u)$ is the cost function that inverts $V(\mathbf{p}, y)$, it follows that $\partial h_{i} / \partial u=\left(\partial q_{i} / \partial y\right)(\partial C / \partial u)$. Hence, using (5), the restrictions in (4) can be stated as

$$
\frac{\partial h_{i}(\mathbf{p}, V(\mathbf{p}, y))}{\partial p_{k}}=\mu_{k}(\mathbf{p}, y) \frac{\partial q_{i}(\mathbf{p}, y)}{\partial y} \frac{\partial q_{k}(\mathbf{p}, y)}{\partial y}
$$

for all $i \in I_{s}$ and $k \in I^{c}$, where $\mu_{k}=[(\partial \Xi /$ $\left.\left.\partial p_{k}\right)(\partial C / \partial u)^{2}\right]\left[(\partial \Xi / \partial u)\left(\partial h_{k} / \partial u\right)\right]^{-1}$, evaluated at 
$u=V(\mathbf{p}, y)$. Note that $\mu_{k}$ depends on which $q_{k}$ in $I^{c}$ one is considering, but not on which $q_{i}$ in $I_{s}$ is being considered. Somewhat loosely, we can conclude from (6) that the asymmetric separability restrictions reduce to those of symmetric separability if each good in $I^{c}$ is interpreted as a separable group.

Given the above, explicitly consider the case in which the first $t$ groups in the partition $\hat{I}$ contain only one good each, such that the separable utility function can be written as

$$
U(\mathbf{q})=U^{0}\left[q_{1}, \ldots, q_{t}, U^{t+1}\left(\mathbf{q}^{t+1}\right), \ldots, U^{S}\left(\mathbf{q}^{S}\right)\right] .
$$

Then, the combination of the results from Goldman and Uzawa, and Blackorby, Davidson, and Schworm can be stated as follows. If we take any two goods $(i, j) \in I_{g}$ and any two goods $(m$, $k) \in I_{s}(i=j$ or $m=k$ is possible), for any two groups $g \neq s$, it follows that the substitution terms between goods belonging to different groups are proportional to the respective income terms:

$$
\frac{\frac{\partial h_{i}(\mathbf{p}, V(\mathbf{p}, y))}{\partial p_{k}}}{\frac{\partial h_{j}(\mathbf{p}, V(\mathbf{p}, y))}{\partial p_{m}}}=\frac{\frac{\partial q_{i}(\mathbf{p}, y)}{\partial y}}{\frac{\partial q_{j}(\mathbf{p}, y)}{\partial y}} \frac{\partial q_{k}(\mathbf{p}, y)}{\partial y} \frac{\partial q_{m}(\mathbf{p}, y)}{\partial y} .
$$

Because (8) is an alternative representation of the necessary and sufficient conditions, it summarizes the relevant empirical restrictions of direct weak separability. ${ }^{1}$ It can be expressed in convenient elasticity form as follows. Let $\sigma_{i j}$ denote the Allen-Uzawa elasticity of substitution between goods $i$ and $j$, such that $\sigma_{i j} \equiv \eta_{i j} / w_{j}$, where $\eta_{i j} \equiv\left(\partial h_{i} / \partial p_{j}\right)\left(p_{j} / q_{i}\right)$ is the compensated cross-price elasticity and $w_{j} \equiv p_{j} q_{j} / y$ is the expenditure share on good $j$. Also, define income elasticities as $\epsilon_{i} \equiv\left(\partial q_{i} / \partial y\right)\left(y / q_{i}\right)$. Then, the restrictions in (8) can be expressed as

$$
\frac{\sigma_{i k}}{\sigma_{j m}}=\frac{\epsilon_{i} \epsilon_{k}}{\epsilon_{j} \epsilon_{m}}
$$

for all $(i, j) \in I_{g}$ and $(m, k) \in I_{s}$, for all $g \neq s$.

\footnotetext{
${ }^{1}$ Our approach here is geared toward econometric applications of separability. Alternatively, one could follow Varian's nonparametric approach to consumer demand based on revealed preferences. A necessary condition for weak separability is that the goods in the separable group satisfy the generalized axiom of revealed preferences (GARP). Sufficient conditions require that the data satisfy both GARP and the Afriat inequalities. Although such conditions can be readily checked, it is not clear how violations ought to be interpreted in a probabilistic sense. Also, Barnett and Choi argue that the use of the sufficient conditions may bias the nonparametric test toward rejection of separability.
}

Equation (9) defines a set of restrictions that can be maintained in any of the commonly used demand systems, or subjected to a statistical test. Note that if the subutility functions are homothetic, then $\epsilon_{i}=\epsilon_{j}$ and $\epsilon_{k}=\epsilon_{m}$, ${ }^{2}$ and the restrictions in (9) reduce to $\sigma_{i k}=\sigma_{j m}$. This is essentially the result obtained by Blackorby and Russell (1976), who proved that $\sigma_{i k}=\sigma_{j k}$ for $(i, j) \in I_{s}$ and $k \notin I_{s}$ (they considered the case of asymmetric weak separability). ${ }^{3}$

To implement the restrictions in (9) for the purpose of testing for separability, it is important to keep track of the number of restrictions that are implied by the assumed separable structure. If $n$ is the total number of goods, there are a total of $1 / 2 n(n-1)$ cross-substitution terms $\partial h_{i} / \partial p_{k}(i \neq k)$. If $n_{s}$ is the number of goods belonging to the $s$ th group $(s=1,2, \ldots, S)$, then there are $1 / 2\left[\Sigma_{s} n_{s}\left(n_{s}-1\right)\right]$ within-group cross-substitution terms. Taking the difference between these two quantities yields the number of substitution terms, say $n_{0}$, that pertain to goods belonging to different groups. Moreover, there will be

$$
\left(\begin{array}{l}
S \\
2
\end{array}\right) \equiv n_{\mu}
$$

proportionality coefficients $\mu$ which completely identify the $n_{0}$ cross-substitution terms given the income effects. Hence, $n_{\mu}=1 / 2 S(S-1)$, and the number $n_{R} \equiv\left(n_{0}-n_{\mu}\right)$ of nonredundant restrictions implied by equation (9) is ${ }^{4}$

(10)

$n_{R}=\frac{1}{2}\left[n(n-1)-\sum_{s=1}^{s} n_{s}\left(n_{s}-1\right)-S(S-1)\right]$

\section{Homothetic Separability}

In addition to justifying conditional demand systems, separability is useful in demand analysis because it can rationalize commodity aggrega-

\footnotetext{
${ }^{2}$ Note that these elasticities need not equal unity unless the utility function $U^{0}(\cdot)$ is itself homothetic.

${ }^{3}$ For the case of nonhomothetic asymmetric weak separability, the restrictions in (9) become $\sigma_{i k} \epsilon_{j}=\sigma_{j k} \epsilon_{i}$ and can be expressed in convenient form by using compensated elasticities or Marshallian elasticities as well. In particular, equivalent expressions are $\eta_{i k}$ $\epsilon_{j}=\eta_{j k} \epsilon_{i}$, or $\epsilon_{i k} \epsilon_{j}=\epsilon_{j k} \epsilon_{i}$, where $\epsilon_{i j} \equiv\left(\partial q_{i} / \partial p_{j}\right)\left(p_{j} / q_{i}\right)$ are Marshallian elasticities.

${ }^{4}$ In a similar fashion, Theil (1976, pp. 68-69) discusses the number of unconstrained parameters under blockwise dependence (symmetric weak separability). Note that if more complex separability trees are considered (see below), the formula in (10) needs to be applied in a suitably recursive fashion.
} 
tion. Clearly, direct weak separability means that, from the consumer point of view, goods belonging to a subutility function can be "aggregated" in a composite commodity. However, if we are to aggregate across commodities for the purpose of modeling demand, we also need a consistent price index for the separable group of goods. For this result, it is typically required that the subutility functions of the goods being aggregated be homothetic. Hence, when the focus is to test alternative commodity aggregates, as in Eales and Unnevehr, the hypothesis of interest is that of homothetic weak separability. A homothetic subutility function $U^{s}\left(\mathbf{q}^{s}\right)$ with $n_{s}$ goods implies that $\epsilon_{i}=\epsilon_{j}$ for all $(i, j) \in I_{s}$, and entails $\left(n_{s}-1\right)$ additional restrictions.

\section{Separability Trees}

By putting further structure on the utility function, one can entertain complex separability hypotheses that put more structure on the way goods enter the utility function. An example is offered by the following utility function:

$$
\begin{aligned}
& \text { (11) } U(\mathbf{q})= \\
& U^{0}\left[q_{1}, U^{A}\left(q_{2}, U^{B}\left[q_{3}, U^{C}\left(q_{4}, q_{5}\right), U^{D}\left(q_{6}, q_{7}\right)\right]\right)\right] .
\end{aligned}
$$

To define the set of necessary and sufficient restrictions, one needs to determine the number of proportionality coefficients $\mu$. The fact that $q_{1}$ and $U^{A}$ form two separable groups in the utility function $U^{0}(\cdot)$ requires one proportionality coefficient. Similarly, the fact that $q_{2}$ and $U^{B}$ form two separable groups within $U^{A}(\cdot)$ gives another proportionality coefficient. Finally, $q_{3}, U^{C}$, and $U^{D}$ form three separable partitions within $U^{B}(\cdot)$ and thus entail three additional proportionality coefficients. Hence, we have a total of five $\mu$ proportionality coefficients. To see how many cross-substitution terms need to be constrained to be consistent with the structure of (11), note that the separability of $\left(q_{1}, U^{A}\right)$ in $U^{0}(\cdot)$ entails six cross-substitution terms pertaining to goods belonging to different groups, whereas the separability of $\left(q_{2}, U^{B}\right)$ in $U^{A}(\cdot)$ and the separability of $\left(q_{3}, U^{C}, U^{D}\right)$ in $U^{B}(\cdot)$ entail five and eight such terms, respectively. Hence, for this example, $n_{S}=19$ and $n_{\mu}=5$, which means that there are 14 independent restrictions implied by the structure in $(11) .^{5}$

\footnotetext{
${ }^{5}$ The utility trees 2 and 3 considered by Eales and Unnevehr have the structure of utility function (11). They report testing only 9 restrictions in each case, however, whereas we have shown that 14 restrictions are required.
}

\section{Separability and Flexible Demand Systems}

The separability conditions summarized by the elasticity restrictions of equation (9) can be maintained or tested upon a parametric specification of a demand system. In what follows, three popular demand systems are considered in turn: the Almost Ideal, the Translog, and the Rotterdam demand systems.

\section{Almost Ideal Demand System}

From a specific parameterization of a PIGLOG cost function, the Almost Ideal demand system (ALIDS) of Deaton and Muellbauer is derived as

$$
w_{i}=\alpha_{i}+\sum_{j=1}^{n} \gamma_{i j} \log \left(p_{j}\right)+\beta_{i} \log \left(\frac{y}{P}\right)
$$

where $\log (\mathrm{P})$ is a translog price index defined as

$$
\begin{aligned}
\log (P)=\alpha_{0} & +\sum_{i=1}^{n} \alpha_{i} \log \left(p_{i}\right) \\
& +\frac{1}{2} \sum_{i=1}^{n} \sum_{j=1}^{n} \gamma_{i j} \log \left(p_{i}\right) \log \left(p_{j}\right) .
\end{aligned}
$$

Homogeneity, adding-up, and symmetry require $\Sigma_{i} \alpha_{i}=1, \Sigma_{j} \gamma_{i j}=\Sigma_{i} \gamma_{i j}=\Sigma_{i} \beta_{i}=0$, and $\gamma_{i j}=$ $\gamma_{j i}$. For this demand model, the elasticities of substitution and the income elasticities can be written as

$$
\begin{gathered}
\sigma_{i k}=\frac{\gamma_{i k}}{w_{i} w_{k}}+\frac{\beta_{i}}{w_{i}} \frac{\beta_{k}}{w_{k}} \log \left(\frac{y}{P}\right)+1 \quad(i \neq k) \\
\epsilon_{i}=1+\frac{\beta_{i}}{w_{i}}
\end{gathered}
$$

Hence, the separability restrictions in (9) can be written as

$$
\begin{aligned}
\gamma_{i k}+w_{i} w_{k}+\beta_{i} \beta_{k} \log \left(\frac{y}{P}\right) \\
\gamma_{j m}+w_{j} w_{m}+\beta_{j} \beta_{m} \log \left(\frac{y}{P}\right) \\
=\frac{\left(w_{i}+\beta_{i}\right)\left(w_{k}+\beta_{k}\right)}{\left(w_{j}+\beta_{j}\right)\left(w_{m}+\beta_{m}\right)} .
\end{aligned}
$$

where $(i, j) \in I_{g}$ and $(k, m) \in I_{s}$ for all $g \neq s .^{6}$

\footnotetext{
${ }^{6}$ These restrictions reduce to those reported by Eales and Unnevehr only if $k=m$, which yields restrictions appropriate for asymmetric separability.
} 
It is evident that the restrictions in (16) involve prices and income [recall that the shares are defined as in (12)]. The restrictions can hold for every possible realization of prices and income (i.e., globally) only under very confining conditions. In particular, the restrictions in (16) will hold globally if $\beta_{i}=\beta_{k}=\beta_{j}=\beta_{m}=0$ and $\gamma_{i k}=\gamma_{j m}=0$. These parametric restrictions have the unwanted implication of homotheticity for the separable groups and, in addition, force the income elasticities of goods in the separable group to equal unity (which is not necessary even under homothetic separability). The restrictiveness of such implications make the global separability restrictions totally undesirable for the purpose of maintaining or testing separability. In other words, the ALIDS model is separabilityinflexible, a feature of a wide class of flexible functional forms (Blackorby, Primont, and Russell 1977).

To overcome this serious limitation, one may resort to different parametric specifications of demand. ${ }^{7}$ Alternatively, if one wants to stick to the ALIDS specification, it may be of some interest to consider the separability restrictions at a point only, as suggested by Denny and Fuss, and by Jorgenson and Lau. ${ }^{8}$ If the point of interest is the mean of the explanatory variables, it is convenient to scale all prices and income to equal unity at the mean. At such a point, then, $w_{i}=\alpha_{i}-\beta_{i} \alpha_{0}$ and $\log (y / P)=-\alpha_{0}$, so the restrictions in (16) can be written as

$$
\begin{array}{r}
\frac{\gamma_{i k}+\left(\alpha_{i}-\beta_{i} \alpha_{0}\right)\left(\alpha_{k}-\beta_{k} \alpha_{0}\right)-\beta_{i} \beta_{k} \alpha_{0}}{\gamma_{j m}+\left(\alpha_{j}-\beta_{j} \alpha_{0}\right)\left(\alpha_{m}-\beta_{m} \alpha_{0}\right)-\beta_{j} \beta_{m} \alpha_{0}} \\
=\frac{\left(\alpha_{i}-\beta_{i} \alpha_{0}+\beta_{i}\right)\left(\alpha_{k}-\beta_{k} \alpha_{0}+\beta_{k}\right)}{\left(\alpha_{j}-\beta_{j} \alpha_{0}+\beta_{j}\right)\left(\alpha_{m}-\beta_{m} \alpha_{0}+\beta_{m}\right)}
\end{array}
$$

for all $(i, j) \in I_{g}$ and $(k, m) \in I_{s}$, for all $g \neq s$. Equation (17) defines a set of nonlinear restrictions that involve only the parameters of the ALIDS model, and these restrictions can be either maintained or tested.

Because parameter $\alpha_{0}$ is virtually impossible to estimate in empirical applications, Deaton and Muellbauer suggest fixing $\alpha_{0}$ before estimating all other parameters, and propose a possible cri-

\footnotetext{
${ }^{7}$ For example, two separability-flexible specifications used in production applications are the Generalized Barnett of Diewert and Wales, as formulated by Blackorby, Schworm, and Fisher, and the FAST model of Moschini.

${ }^{8}$ The resulting 'local' test is also referred to as the 'approximate' separability test because it relies on the (local) approximation properties of the flexible functional form used. Relying on such a local test to make inferences about separability has drawn some criticism (see Aizcorbe for a discussion).
}

terion for doing so. The reason for the estimation problem is that the likelihood function typically is extremely flat in $\alpha_{0}$, suggesting the actual choice of $\alpha_{0}$ does not matter for the approximation properties of ALIDS. ${ }^{9}$ Hence, an alternative and particularly useful criterion in our context is to set $\alpha_{0}=0$, in which case the local restrictions in (17) simplify to the ones in (20) below.

Often, a linear version of the ALIDS model is estimated, where the translog aggregator $\log (P)$ is substituted by a price index prior to estimation, say the Stone index $\log \left(P^{*}\right)=\Sigma_{i} w_{i} \log \left(p_{i}\right)$. In such case, scaling prices is very important, over and above the simplifications induced in the local separability restrictions, because the Stone price index is not invariant to the choice of units of measurement (Moschini and Vissa). An appropriate substitution elasticity formula for the linear ALIDS with the Stone price index, consistent with taking the Stone index as given in estimation, is (Chalfant)

$$
\alpha_{i k}=\frac{\gamma_{i k}}{w_{i} w_{k}}+1 \quad(i \neq k)
$$

Hence, the separability restrictions for the linear ALIDS can be expressed as

$$
\frac{\gamma_{i k}+w_{i} w_{k}}{\gamma_{j m}+w_{j} w_{m}}=\frac{\left(w_{i}+\beta_{i}\right)\left(w_{k}+\beta_{k}\right)}{\left(w_{j}+\beta_{j}\right)\left(w_{m}+\beta_{m}\right)} \text {. }
$$

These restrictions are very similar to those of the nonlinear ALIDS model of equation (16). In particular, the local separability restrictions [at mean point $(\mathbf{p}, y)=(1,1)]$ reduce to ${ }^{10}$

$$
\frac{\gamma_{i k}+\alpha_{i} \alpha_{k}}{\gamma_{j m}+\alpha_{j} \alpha_{m}}=\frac{\left(\alpha_{i}+\beta_{i}\right)\left(\alpha_{k}+\beta_{k}\right)}{\left(\alpha_{j}+\beta_{j}\right)\left(\alpha_{m}+\beta_{m}\right)} .
$$

If one were interested in testing for homothetic separability, the global test for weak separability is clearly unchanged, given that it entails homotheticity of the subutility functions. The local test restrictions of (17) or (20), however, must in this case be supplemented by restrictions $\alpha_{i} / \beta_{i}=\alpha_{j} / \beta_{j}$ and $\alpha_{k} / \beta_{k}=\alpha_{m} / \beta_{m}$.

\footnotetext{
${ }^{9}$ In other words, one should expect that computed elasticities are not affected by choice of $\alpha_{0}$ (although the estimated parameters clearly are), a proposition supported by our empirical experience.

${ }^{10}$ Using elasticity formula (14) for the linear ALIDS model is appropriate only if one ensures that the parameters of the linear ALIDS model approximate the parameters of the nonlinear ALIDS model. If prices and income are scaled to equal one at the mean, the estimated parameters of linear ALIDS will approximate the parameters of a nonlinear ALIDS with $\alpha_{0}=0$, in which case the elasticity formulae for nonlinear ALIDS also apply to the linear ALIDS (the problems discussed by Green and Alston do not arise). In such a case, local restrictions (17) and (20) are clearly identical.
} 


\section{Translog Model}

From a translog specification of the indirect utility function, the following demand system can be derived (Christensen, Jorgenson, and Lau):

$$
w_{i}=\frac{\alpha_{i}+\sum_{j=1}^{n} \gamma_{i j} \log \left(\frac{p_{j}}{y}\right)}{1+\sum_{j=1}^{n} \sum_{k=1}^{n} \gamma_{k j} \log \left(\frac{p_{k}}{y}\right)}
$$

where normalization $\Sigma_{j} \alpha_{j}=1$ is adopted. ${ }^{11}$ Homogeneity and adding-up are satisfied by (21), while symmetry requires $\gamma_{i j}=\gamma_{j i}$. Moreover, it is convenient to impose the additional normalization $\Sigma_{i} \Sigma_{j} \gamma_{i j}=0$, so that the translog can be aggregated exactly across consumers. ${ }^{12}$ With this aggregation condition, and defining parameter $\beta_{i} \equiv \Sigma_{j} \gamma_{i j}$, the translog demand model generates substitution and income elasticities that can be written as

$$
\begin{array}{r}
\sigma_{i j}=\frac{\frac{\gamma_{i j}}{w_{i} w_{j}}-\frac{\beta_{i}}{w_{i}}-\frac{\beta_{j}}{w_{j}}}{1+\sum_{r=1}^{n} \beta_{r} \log \left(p_{r}\right)}+1 \\
\epsilon_{i}=1-\frac{\beta_{i}}{\alpha_{i}+\sum_{r=1}^{n} \gamma_{i r} \log \left(\frac{p_{r}}{y}\right)} .
\end{array}
$$

Hence, the separability restrictions in (9) can be expressed as

$$
\begin{aligned}
& \frac{\gamma_{i k}-w_{i} \beta_{k}-w_{k} \beta_{i}+w_{i} w_{k}\left(1+\sum_{r} \beta_{r} \log \left(p_{r}\right)\right)}{\gamma_{j m}-w_{j} \beta_{m}-w_{m} \beta_{j}+w_{j} w_{m}\left(1+\sum_{r} \beta_{r} \log \left(p_{r}\right)\right)}= \\
& \frac{\left(\alpha_{i}+\sum_{r} \gamma_{i r} \log \left(\frac{p_{r}}{y}\right)-\beta_{i}\right)\left(\alpha_{k}+\sum_{r} \gamma_{k r} \log \left(\frac{p_{r}}{y}\right)-\beta_{k}\right)}{\left(\alpha_{j}+\sum_{r} \gamma_{j r} \log \left(\frac{p_{r}}{y}\right)-\beta_{j}\right)\left(\alpha_{m}+\sum_{r} \gamma_{m r} \log \left(\frac{p_{r}}{y}\right)-\beta_{m}\right)}
\end{aligned}
$$

for all $(i, j) \in I_{g}$ and $(k, m) \in I_{s}$, for all $g \neq s$.

\footnotetext{
"A normalization is required here, although the choice is arbitrary (Christensen, Jorgenson, and Lau). The normalization adopted is convenient in that $w_{i}=\alpha_{i}$ at point $(\mathbf{p}, y)=(1,1)$ (i.e., the mean point given our scaling convention).

${ }^{12}$ With this parametric restriction, the Translog model becomes a member of the generalized Gorman polar form family of preferences, similar to the ALIDS (Lewbel).
}

As for the case of ALIDS, the restrictions of (24) can be satisfied globally only under very strong conditions. Specifically, one needs $\beta_{i}=$ $\beta_{k}=\beta_{j}=\beta_{m}=0$ and $\gamma_{i k}=\gamma_{j m}=0$, implying that the separable group aggregators are homothetic and the unconditional income elasticities of the goods involved are equal to one. Such undesirable global conditions again suggest considering the separability restrictions at a point. If prices and income have been scaled to have a mean equal to unity, the separability restrictions of (24) at the mean point can be expressed as

$$
\begin{aligned}
\frac{\gamma_{i k}-\alpha_{i} \beta_{k}-\alpha_{k} \beta_{i}}{\gamma_{j m}-\alpha_{j} \beta_{m}-\alpha_{i} \alpha_{k} \beta_{j}}+\alpha_{j} \alpha_{m} \\
=\frac{\left(\alpha_{i}-\beta_{i}\right)\left(\alpha_{k}-\beta_{k}\right)}{\left(\alpha_{j}-\beta_{j}\right)\left(\alpha_{m}-\beta_{m}\right)}
\end{aligned}
$$

As for the ALIDS model, if one wants to test for homothetic separability, the global test for weak separability is unchanged. The local test restrictions of $(25)$, on the other hand, would have to be supplemented by restrictions $\alpha_{i} / \beta_{i}=$ $\alpha_{j} / \beta_{j}$ and $\alpha_{k} / \beta_{k}=\alpha_{m} / \beta_{m}$.

\section{Rotterdam Model}

From a differential approximation to the Marshallian demand functions, the absolute price version of the Rotterdam model is written as (Theil 1980)

$$
w_{i} d \log \left(q_{i}\right)=\theta_{i} d \log (Q)+\sum_{j=1}^{n} \pi_{i j} d \log \left(p_{j}\right)
$$

where $d \log (Q)=\Sigma_{j} w_{j} d \log \left(q_{j}\right)$ is the Divisia volume index. Homogeneity, adding-up, and symmetry require $\Sigma_{j} \theta_{j}=1, \Sigma_{j} \pi_{i j}=\Sigma_{i} \pi_{i j}=0$, and $\pi_{i j}=\pi_{j i}$. Because of the parameterization chosen, substitution and income elasticities can be written as

$$
\begin{aligned}
\sigma_{i j} & =\frac{\pi_{i j}}{w_{i} w_{j}} \\
\epsilon_{i} & =\frac{\theta_{i}}{w_{i}} .
\end{aligned}
$$

Hence the separability restrictions of (9) can here be expressed as

$$
\frac{\pi_{i k}}{\pi_{j m}}=\frac{\theta_{i} \theta_{k}}{\theta_{j} \theta_{m}}
$$

for all $(i, j) \in I_{g}$ and $(k, m) \in I_{s}$, for all $g \neq s$. 
The remarkable feature of this result is that the separability restrictions will hold not only locally (at a point), but also globally, without any further restriction. This sets the Rotterdam model apart from other functional forms, such as the Translog and ALIDS analyzed above, and would seem to suggest that the Rotterdam model is separability-flexible for the purpose of modeling weak separability.

It should be understood, however, that the Rotterdam model provides a different type of approximation to the underlying demand system. Translog and ALIDS are approximations in the variable space. Although they are best interpreted as approximating functions, they could represent preferences exactly, at least over a region of parameters satisfying regularity conditions. On the other hand, the Rotterdam model is best interpreted as an approximation in the parameter space. From an exact differential representation to an arbitrary demand system, the approximation is introduced by assuming that the Slutsky terms $\pi_{i j}$ and the real income terms $\theta_{i}$ are constant (and, in empirical application, log differentials are approximated by finite log differences). It follows that the Rotterdam model cannot be considered an exact representation of preferences unless very strong conditions are imposed. ${ }^{13}$ Hence, satisfying the separability restrictions globally, with the minimum number of parametric restrictions, does not make the Rotterdam model a separability-flexible representation of preferences.

Regardless of the interpretation issues just discussed, equation (29) yields the simplest parametric representation of separability restrictions among the three demand systems considered. This simplicity has obvious attraction for empirical applications. The parametric convenience of the Rotterdam model for direct weak separability does not necessarily carry over to other types of separability, a point clearly illustrated by the case of homothetic weak separability. Homotheticity for the subutility function $U^{s}\left(\mathbf{q}^{s}\right)$ implies that $\epsilon_{i}=\epsilon_{j}$ for all $(i, j) \in I_{s}$. In view of (28), the additional restrictions that must be satisfied are

$$
\frac{\theta_{i}}{w_{i}}=\frac{\theta_{j}}{w_{j}} .
$$

It is not difficult to test or impose these addi-

\footnotetext{
${ }^{13}$ This is the so-called McFadden critique and is discussed by Theil (1975, pp. 101-05.) Of course, such a result does not detract at all from the usefulness of the Rotterdam model as a flexible approximation to an unknown demand system (Barnett 1979a; Mountain).
}

tional restrictions at a point. However, these restrictions will hold globally only under unwanted strong conditions. In particular, the shares involved would need to be constant, which for the absolute-price version of the Rotterdam model in (26) requires $w_{i}=\theta_{i}, \pi_{i j}=\theta_{i} \theta_{j}$ [for $i \neq j$, $\left.(i, j) \in I_{s}\right]$, and $\pi_{i i}=\theta_{i}\left(\theta_{i}-1\right) .^{14}$

\section{Application to a U.S. Demand System}

To illustrate maintaining and testing separability restrictions, we apply the Rotterdam model to a U.S. data set emphasizing food demand, with particular attention to the meat group, reported in Blanciforti, Green, and King (henceforth BGK). ${ }^{15}$ More exactly, we specify a seven-good demand system with the following goods: $q_{1}=$ nonfood; $q_{2}=$ fruits and vegetables; $q_{3}=$ cereals and bakery products; $q_{4}=$ miscellaneous foods; $q_{5}=$ beef and veal; $q_{6}=$ pork; and, $q_{7}$ $=$ poultry and fish. Fish and meat products are as in BGK's tables 5.A.6 and 5.A.8 (fish and poultry are aggregated by a Divisia index); similarly, the other three food aggregates are from BGK's tables 5.A.10 and 5.A.12. Finally, the nonfood commodity is a Divisia aggregate of ten nonfood categories reported in BGK's tables 5.A. 1 and 5.A.3. ${ }^{16}$ The period covered by these data is $1947-78$.

We consider three different types of weakly separable structures, all of which are tested against the unrestricted utility function $U(\mathbf{q})$. First, we postulate that food commodities are weakly separable from nonfood commodities, such that the utility function can be written as

$$
U=U^{0}\left[q_{1}, f\left(q_{2}, q_{3}, q_{4}, q_{5}, q_{6}, q_{7}\right)\right]
$$

Following the earlier discussion, it is verified that this structure entails 5 nonredundant restrictions relative to the unrestricted utility $U(\mathbf{q})$. For the Rotterdam specification, these nonredundant restrictions can be represented as:

\footnotetext{
${ }^{14}$ Hence, for the shares of goods belonging to a homothetically separable group to be constant, the parameterization of the Rotterdam model chosen here requires (for these goods) $\epsilon_{i}=1, \epsilon_{i i}=-1$, and $\epsilon_{i j}=0$.

${ }^{15}$ One reviewer correctly pointed out that whereas the theory discussed earlier pertains to an individual consumer, our application (similar to most such applications) relies on aggregate data. The three models considered in this paper can, in principle, be consistent with the notion of a representative consumer. However, a rigorous analysis of how aggregation across consumers may interact with separability restrictions is beyond the scope of the present article.

${ }^{16}$ Total expenditure was constructed as the sum of the expenditures on these aggregates.
} 


$$
\begin{aligned}
& \frac{\pi_{12}}{\pi_{17}}=\frac{\theta_{2}}{\theta_{7}}, \quad \frac{\pi_{13}}{\pi_{17}}=\frac{\theta_{3}}{\theta_{7}}, \\
& \frac{\pi_{14}}{\pi_{17}}=\frac{\theta_{4}}{\theta_{7}}, \quad \frac{\pi_{15}}{\pi_{17}}=\frac{\theta_{5}}{\theta_{7}}, \\
& \frac{\pi_{16}}{\pi_{17}}=\frac{\theta_{6}}{\theta_{7}} .
\end{aligned}
$$

The second hypothesis is that meat commodities are separable from other foods within the food group, in addition to food being separable from nonfood. In other words, the utility function is written as

$$
U=U^{0}\left(q_{1}, f\left[q_{2}, q_{3}, q_{4}, m\left(q_{5}, q_{6}, q_{7}\right)\right]\right) .
$$

This structure entails 11 nonredundant restrictions, which for the Rotterdam specification can be represented as (32) plus the following six restrictions:

$$
\begin{aligned}
& \frac{\pi_{25}}{\pi_{27}}=\frac{\theta_{5}}{\theta_{7}}, \quad \frac{\pi_{26}}{\pi_{27}}=\frac{\theta_{6}}{\theta_{7}}, \\
& \frac{\pi_{35}}{\pi_{37}}=\frac{\theta_{5}}{\theta_{7}}, \quad \frac{\pi_{36}}{\pi_{37}}=\frac{\theta_{6}}{\theta_{7}}, \\
& \frac{\pi_{45}}{\pi_{47}}=\frac{\theta_{5}}{\theta_{7}}, \quad \frac{\pi_{46}}{\pi_{47}}=\frac{\theta_{6}}{\theta_{7}} .
\end{aligned}
$$

The third hypothesis is that within food we have two (symmetrically) separable groups, meat and nonmeat, where food is still separable from nonfood. That is

$$
U=U^{0}\left(q_{1}, f\left[v\left(q_{2}, q_{3}, q_{4}\right), m\left(q_{5}, q_{6}, q_{7}\right)\right]\right) .
$$

Structure (35) entails 13 nonredundant restrictions. For the Rotterdam specification, these restrictions can be represented as (32) plus (34) and the following two restrictions:

$$
\frac{\pi_{27}}{\pi_{47}}=\frac{\theta_{2}}{\theta_{4}}, \quad \frac{\pi_{37}}{\pi_{47}}=\frac{\theta_{3}}{\theta_{4}} .
$$

For purpose of estimation, the demand model (which is nonlinear in the separable cases) can be written as a system of seemingly unrelated regressions:

$$
\mathbf{Y}_{t}=F\left(\mathbf{Z}_{t}, \boldsymbol{\beta}\right)+\mathbf{e}_{t} \quad t=1,2, \ldots, T
$$

where $\mathbf{Y}_{t}$ is a vector of $w_{i} d \log \left(q_{i}\right)$ at time $t, \boldsymbol{\beta}$ is the vector of all coefficients to be estimated, $\mathbf{Z}_{t}$ is the vector of the corresponding exogenous variables at time $t$, and $\mathbf{e}_{t}$ is a vector of error terms. Errors are assumed contemporaneously correlated but serially uncorrelated; in other words, $E\left(\mathbf{e}_{t}\right)=\mathbf{0}, E\left(\mathbf{e}_{t} \mathbf{e}_{t}^{\prime}\right)=\boldsymbol{\Omega}$ for all $t$, and $E\left(\mathbf{e}_{t} \mathbf{e}_{s}\right)=\mathbf{0}$ for $t \neq s .{ }^{17}$ Assuming that the $\mathbf{e}_{t}$ 's are multinormally distributed, maximum likelihood estimation is performed, yielding estimators that are consistent, asymptotically normal, asymptotically efficient, and invariant to which equation is omitted. To implement the maximum likelihood estimator, we rely on an algorithm implemented in TSP version 4.2A.

\section{Testing Separability with the Wald Test}

The separability restrictions to be tested are nonlinear parametric restrictions, say $g(\boldsymbol{\beta})=\mathbf{0}$ where $g(\cdot)$ is vector-valued. To test such parametric restrictions, it is common to use the Wald test, which requires estimation of the unrestricted model only. The Wald test is computationally appealing because with nonlinear restrictions (such as those of separability) the formulation of the restricted model is somewhat cumbersome, especially when one has to deal simultaneously with other linear restrictions (homogeneity and symmetry) in a multi-equation setting. Unfortunately, this procedure has a severe drawback in the context of separability tests because, in finite samples, the numerical value of the Wald test lacks invariance when nonlinear restrictions are involved (Dagenais and Dufour). ${ }^{18}$

The Wald test for separability lacks invariance with respect to two computational procedures. First, it is not invariant with respect to the parametric formulation of the null hypothesis (Gregory and Veall). To illustrate, if $g_{r}(\boldsymbol{\beta})$ denotes the $r$ th restriction to be tested, two (among many) equivalent representations of the separability restrictions are

$$
\begin{aligned}
& g_{r}(\boldsymbol{\beta})=\frac{\pi_{i k}}{\pi_{j k}}-\frac{\theta_{i}}{\theta_{j}}=0 \\
& g_{r}(\boldsymbol{\beta})=\pi_{i k} \theta_{j}-\pi_{j k} \theta_{i}=0 .
\end{aligned}
$$

In addition, the Wald test is not invariant with respect to the choice of which nonredundant separability restrictions to use to represent the

\footnotetext{
${ }^{17}$ One of the shares is omitted because of the well known singularity problem of share equation systems.

${ }^{18}$ Essentially, this is because the Wald test is based on a linearization of the nonlinear restrictions, and the linearizations differ depending on the way in which the nonlinear restrictions are represented.
} 
null hypothesis. To illustrate, (32) represents five nonredundant restrictions implied by (31). However, there are many equivalent ways of expressing these five nonredundant restrictions. For example, in (32) we have used $\pi_{17}$ and $\theta_{7}$ in the denominator, but any $\pi_{1 j}$ and $\theta_{j}(j=2$, $\ldots, 7)$ could have been selected for this role.

Results for the three structures discussed above, not reported here for space reasons, indicate that the noninvariance of the Wald test is a real problem. One can readily generate wildly different values for Wald separability tests, showing that "obtaining any Wald Statistics you want," as Lafontaine and White put it, is quite easy.

\section{Testing Separability with the Likelihood Ratio Test}

An obvious test which is invariant for the nonlinear restrictions of separability is the likelihood ratio $L R \equiv 2[L(\hat{\boldsymbol{\beta}})-L(\tilde{\boldsymbol{\beta}})]$, where $L(\cdot)$ denotes the maximized value of the log-likelihood function, $\hat{\boldsymbol{\beta}}$ is the unrestricted estimator of the parameter vector, and $\tilde{\boldsymbol{\beta}}$ is the estimated parameter vector under the separability restrictions. A possible problem with $L R$ in our context is the size of the test. Following Laitinen and Meisner, it is known that tests of restrictions in large demand systems are biased toward rejection. The reason for the bias is that one must use an estimate of the contemporaneous covariance matrix instead of the true matrix. This suggests the need for a size-correction.

There is no accepted way of size-correcting the $L R$ test. The method proposed by Italianer has been found to work very well for linear restrictions (De Boer and Harkema), and is a possibility here. This method relies on a 'corrected' likelihood ratio $L R_{0}$, defined as:

$$
L R_{0}=L R\left[\frac{M T-\frac{1}{2}\left(N_{U}+N_{R}\right)-\frac{1}{2} M(M+1)}{M T}\right]
$$

where $M$ is the number of equations, $T$ is the number of time series observations (hence, there are $M T$ effective observations in the sample and $1 / 2 M(M+1)$ terms in the covariance matrix), $N_{U}$ is the number of parameters of the unrestricted model, and $N_{R}$ is the number of parameters in the restricted (separable) model.

Because the available evidence concerning this size-correction deals with linear restrictions (symmetry and homogeneity), it is necessary to verify that such a correction is appropriate for the nonlinear restrictions of interest here. To this end, we carry out a small Monte Carlo simulation as follows. For each of the three separable structures, we take the estimated restricted (separable) model as the true data-generating process. We generate 500 artificial samples of the left-hand-side vectors by adding randomly generated multinormal errors (using the estimated covariance matrix) to the fitted values of the dependent variables. For each of these 500 samples, we estimated both the unrestricted and the restricted model, then computed the $L R$ statistic and the corrected $L R_{0}$ statistic.

Given that the hypothesis being tested is true by construction, the number of rejections from this exercise should equal (in percent terms) the significance level of the test. Table 1 reports the percentage of rejections of the (true) null hypothesis of separability, using both the nominal $L R$ statistics and the corrected $L R_{0}$ statistics. It is clear that $L R$ leads to overrejection of the hypothesis of separability. The correction suggested by Italianer seems to work well, leading to rejection rates very close to the significance levels. Hence, we can rely on this correction to test our three separable structures. Table 2 reports the likelihood ratio tests of the three hypotheses of interest. Using the corrected $L R_{0}$ test, none of the three separable models can be rejected.

To show what the alternative separability assumptions imply for elasticities, table 3 reports the estimated Allen-Uzawa elasticities of substitution for the unrestricted model and for the three separable models. ${ }^{19}$ This table illustrates

Table 1. Size Correction for Likelihood Ratio Tests of Separability

\begin{tabular}{lccc}
\hline & \multicolumn{3}{c}{$\begin{array}{c}\text { Percent rejection of true } \\
\text { null hypothesis }\end{array}$} \\
\cline { 2 - 4 } & \multicolumn{3}{c}{ significance level of test } \\
& $10 \%$ & $5 \%$ & $1 \%$ \\
\hline Using $L R$ & & & \\
Model (31) & 19.6 & 12.6 & 5.0 \\
Model (33) & 25.8 & 16.4 & 6.4 \\
Model (35) & 26.8 & 15.6 & 5.6 \\
Using $L R_{0}$ & & & \\
Model (31) & 10.2 & 5.4 & 1.4 \\
Model (33) & 9.6 & 5.0 & 1.6 \\
Model (35) & 8.4 & 5.4 & 1.4 \\
\hline
\end{tabular}

Note: Simulation results based on 500 replications.

\footnotetext{
${ }^{19}$ For space reasons, we cannot report more estimation results It is worth noting that all estimated models satisfied concavity of the Slutsky matrix.
} 
Table 2. Likelihood Ratio Tests of Separability

\begin{tabular}{lccc}
\hline & \multicolumn{3}{c}{$\begin{array}{c}\text { Separable structure } \\
\text { of equation: }\end{array}$} \\
& $(31)$ & $(33)$ & $(35)$ \\
\hline$L R$ & 9.31 & 19.15 & 19.76 \\
$L R_{0}$ & 7.03 & 14.77 & 15.35 \\
number of restrictions & 5 & 11 & 13 \\
$\chi_{0.05}^{2}$ & 11.07 & 19.68 & 22.36 \\
\hline
\end{tabular}

the restrictions that separability puts on some of the off-diagonal elements, as discussed earlier. The diagonal elements of these matrices of elasticities seem relatively unaffected. For the last two separable models, maintaining separability causes all off-diagonal elasticities to be positive, reinforcing the predominant relation displayed by the unrestricted model. One should keep in mind, however, that the use of Allen-Uzawa elasticities of substitution to characterize substitutability is highly questionable because, as shown by Blackorby and Russell (1989), such elasticities have no natural interpretation or meaning when more than two goods are involved.

Alternatively, the implications of the separability assumptions can be illustrated by the Marshallian elasticities of table 4. Some of the cross-elasticities are clearly affected by the assumption of separability, especially the elasticities of food items relative to the price of nonfood. In a way, both the magnitude and sign of such elasticities are more appealing for the separable models than for the unrestricted model. Own-price elasticities seem rather robust, and for all separable structures they are fairly similar to those of the unrestricted model. Income elasticities are affected a bit more by separability,

Table 3. Allen-Uzawa Elasticities of Substitution

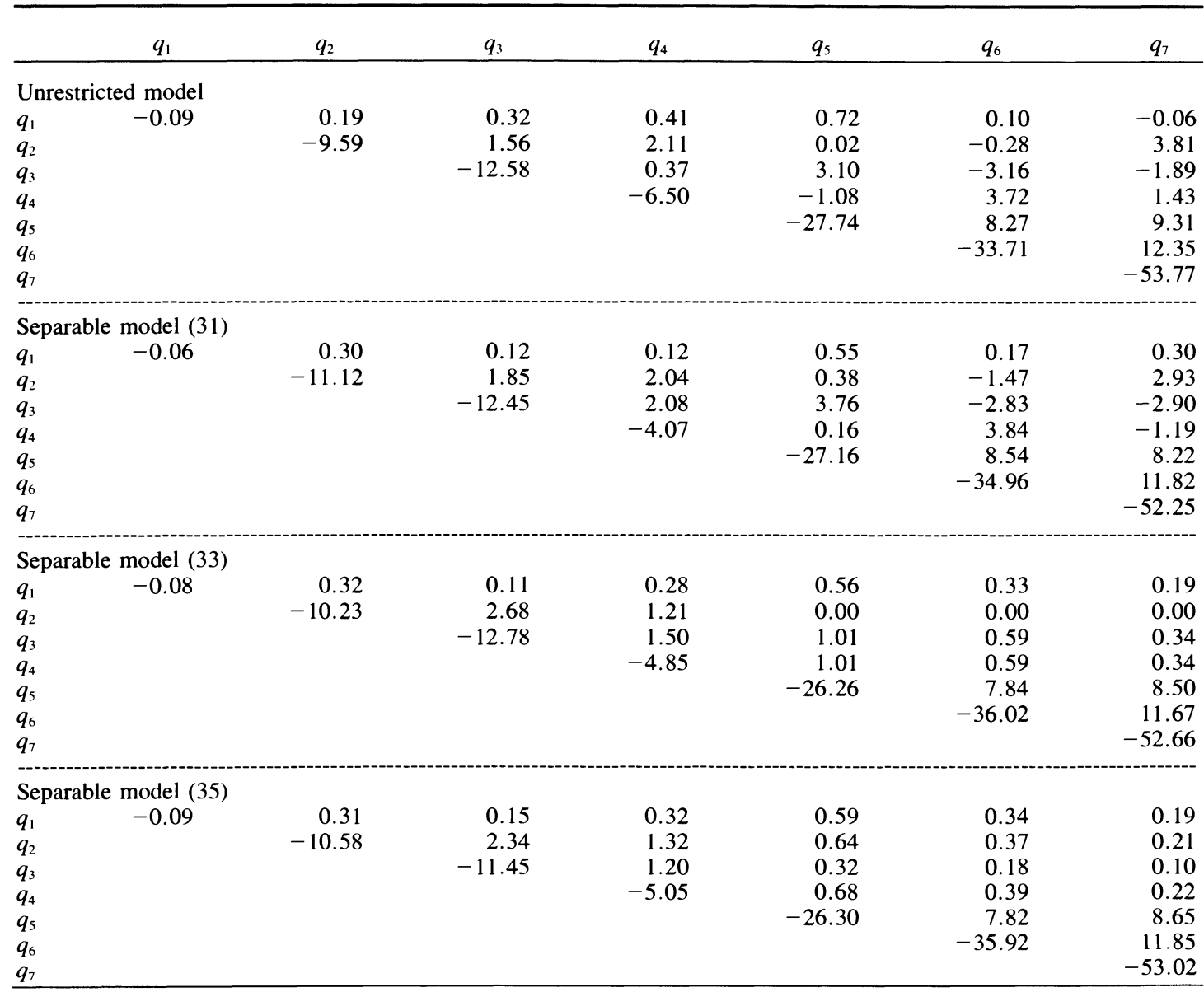

Note: see text for symbols' definition. 
Table 4. Marshallian Elasticities at the Mean

\begin{tabular}{|c|c|c|c|c|c|c|c|c|}
\hline & $p_{1}$ & $p_{2}$ & $p_{3}$ & $p_{4}$ & $p_{5}$ & $p_{6}$ & $p_{7}$ & $y$ \\
\hline \multicolumn{9}{|c|}{ Unrestricted model } \\
\hline$q_{1}$ & -1.03 & -0.04 & -0.02 & -0.06 & -0.02 & -0.02 & -0.02 & 1.20 \\
\hline$q_{2}$ & -0.18 & -0.41 & 0.03 & 0.13 & -0.01 & -0.01 & 0.04 & 0.42 \\
\hline$q_{3}$ & 0.22 & 0.06 & -0.35 & 0.02 & 0.10 & -0.06 & -0.02 & 0.03 \\
\hline$q_{4}$ & 0.36 & 0.09 & 0.01 & -0.48 & -0.03 & 0.07 & 0.02 & -0.04 \\
\hline$q_{5}$ & 0.12 & -0.02 & 0.07 & -0.12 & -0.88 & 0.15 & 0.11 & 0.57 \\
\hline$q_{6}$ & -0.13 & -0.02 & -0.10 & 0.26 & 0.25 & -0.68 & 0.16 & 0.26 \\
\hline$q_{7}$ & -0.48 & 0.13 & -0.07 & 0.07 & 0.27 & 0.23 & -0.70 & 0.55 \\
\hline \multicolumn{9}{|c|}{ Separable model (31) } \\
\hline$q_{1}$ & -0.99 & -0.04 & -0.03 & -0.08 & -0.02 & -0.02 & -0.01 & 1.19 \\
\hline$q_{2}$ & -0.05 & -0.47 & 0.04 & 0.13 & 0.00 & -0.04 & 0.03 & 0.35 \\
\hline$q_{3}$ & -0.02 & 0.07 & -0.35 & 0.14 & 0.11 & -0.06 & -0.04 & 0.14 \\
\hline$q_{4}$ & -0.02 & 0.08 & 0.05 & -0.31 & 0.00 & 0.07 & -0.02 & 0.14 \\
\hline$q_{5}$ & -0.08 & -0.01 & 0.09 & -0.04 & -0.86 & 0.16 & 0.10 & 0.65 \\
\hline$q_{6}$ & -0.03 & -0.07 & -0.08 & 0.27 & 0.26 & -0.70 & 0.15 & 0.20 \\
\hline$q_{7}$ & -0.04 & 0.11 & -0.09 & -0.11 & 0.24 & 0.23 & -0.68 & 0.35 \\
\hline \multicolumn{9}{|c|}{ Separable model (33) } \\
\hline$q_{1}$ & -0.99 & -0.03 & -0.03 & -0.07 & -0.02 & -0.02 & -0.01 & 1.16 \\
\hline$q_{2}$ & -0.06 & -0.44 & 0.06 & 0.06 & -0.01 & -0.01 & -0.01 & 0.39 \\
\hline$q_{3}$ & -0.02 & 0.11 & -0.36 & 0.10 & 0.03 & 0.01 & 0.00 & 0.13 \\
\hline$q_{4}$ & -0.05 & 0.04 & 0.03 & -0.39 & 0.02 & 0.00 & 0.00 & 0.34 \\
\hline$q_{5}$ & -0.10 & -0.03 & 0.01 & 0.02 & -0.84 & 0.14 & 0.10 & 0.68 \\
\hline$q_{6}$ & -0.06 & -0.02 & 0.01 & 0.01 & 0.23 & -0.72 & 0.15 & 0.40 \\
\hline$q_{7}$ & -0.03 & -0.01 & 0.00 & 0.01 & 0.26 & 0.23 & -0.68 & 0.23 \\
\hline \multicolumn{9}{|c|}{ Separable model (35) } \\
\hline$q_{1}$ & -0.99 & -0.04 & -0.03 & -0.06 & -0.02 & -0.02 & -0.01 & 1.16 \\
\hline$q_{2}$ & -0.03 & -0.45 & 0.06 & 0.07 & 0.01 & 0.00 & 0.00 & 0.35 \\
\hline$q_{3}$ & -0.02 & 0.09 & -0.33 & 0.08 & 0.00 & 0.00 & 0.00 & 0.17 \\
\hline$q_{4}$ & -0.03 & 0.04 & 0.02 & -0.40 & 0.01 & 0.00 & 0.00 & 0.36 \\
\hline$q_{5}$ & -0.06 & 0.00 & -0.01 & 0.00 & -0.84 & 0.14 & 0.10 & 0.66 \\
\hline$q_{6}$ & -0.03 & 0.00 & -0.01 & 0.00 & 0.23 & -0.72 & 0.15 & 0.38 \\
\hline$q_{7}$ & -0.02 & 0.00 & 0.00 & 0.00 & 0.26 & 0.23 & -0.69 & 0.22 \\
\hline
\end{tabular}

Note: see text for symbols' definition.

although they are fairly similar across the three separable structures.

\section{Conclusion}

Separability concepts are important in applied demand analysis. Arguably, more efforts should be made to test separability conditions in empirical models and to maintain explicit separable structures in large demand systems. We have reviewed the necessary and sufficient conditions for direct weak separability and homothetic direct weak separability in both symmetric and asymmetric separable structures, casting these conditions in a unified framework suitable for empirical application. Necessary and sufficient parametric restrictions were derived explicitly for three common demand systems: ALIDS, Translog, and Rotterdam. Using the latter model, testing and maintaining separability was illustrated with an empirical application emphasizing U.S. food and meat demand.

Results of the empirical application provide some support for common separability assumptions concerning food and meat products, and would seem to justify the widespread practice of modeling food items and meat products in terms of conditional demand systems. However, if one is concerned with the simultaneity problems discussed by LaFrance, or one wants unconditional elasticities possibly better suited for policy analysis, it may be desirable to maintain separability restrictions within a complete demand model. For such purpose, the approach illustrated in this article provides a consistent use of separability restrictions in empirical demand systems. The Wald test for separability should be avoided because it is not invariant. The likelihood ratio gives a more desirable procedure for 
testing separability, and our results indicate that it is not difficult to correct for the likelihood ratio's tendency to overreject in large demand systems.

\section{[Received May 1992; final revision received May 1993.]}

\section{References}

Aizcorbe, A. M. "A Note on Empirical Tests of Separability and the Approximation View of Functional Forms." South. Econ. J. 59(1992)(2):310-18.

Allen, R. G. B. Mathematical Analysis for Economists. London: MacMillan, 1938.

Alston, J., C. Carter, R. Green, and D. Pick. "Whither Armington Trade Models?" Amer. J. Agr. Econ. 72(May 1990):455-67.

Baccouche, R., and F. Laisney. "Describing the Separability Properties of Empirical Demand Systems." $J$. Appl. Econometrics 6(April 1991):181-206.

Barnett, W. A. "Theoretical Foundations for the Rotterdam Model." Rev. Econ. Stud. 46(January 1979a):109-30. "The Joint Allocation of Leisure and Goods Expenditure." Econometrica 47(May 1979b):539-63.

Barnett, W. A., and S. Choi. "A Monte Carlo Study of Tests of Blockwise Weak Separability." J. Bus. Econ. Stat. 7(July 1989):362-77.

Blackorby, C., R. Davidson, and W. Schworm. "Implicit Separability: Characterization and Implications for Consumer Demands." J. Econ. Theory 55(December 1991):364-99.

Blackorby, C., D. Primont, and R. R. Russell. "On Testing Separability Restrictions with Flexible Functional Forms.” J. Econometrics 5(March 1977):195-209.

- Duality, Separability, and Functional Structure: Theory and Economic Applications (North-Holland, New York, 1978).

Blackorby, C., and R. R. Russell. "Functional Structure and the Allen Partial Elasticities of Substitution: An Application of Duality Theory." Rev. Econ. Stud. 43(June 1976):285-91.

. "Will the Real Elasticity of Substitution Please Stand Up?” Amer. Econ. Rev. 79 (September 1989):882-88.

Blackorby, C., W. Schworm, and T. Fisher. "Testing for the Existence of Input Aggregates in an Economy Production Function." Discussion paper No. 86-26, Department of Economics, University of British Columbia, June 1986.

Blanciforti, L. A., R. D. Green, and G. A. King. U.S. Consumer Behavior Over the Postwar Period: An Almost Ideal Demand System Analysis. Giannini Foundation Monograph No. 40, August 1986.

Byron, R. P. "A Simple Method for Estimating Demand Systems under Separable Utility Assumptions.” Rev. Econ. Stud. 37(April 1970):261-74.

Chalfant, J. A. "A Globally Flexible, Almost Ideal De- mand System.” J. Bus. Econ. Stat. 5(April 1987):23342.

Christensen, L. R., D. W. Jorgenson, and L. J. Lau. "Transcendental Logarithmic Utility Functions." Amer. Econ. Rev. 65(June 1975):367-83.

Dagenais, M. G., and J. M. Dufour. "Invariance, Nonlinear Models, and Asymptotic Tests." Econometrica 59(November 1991):1601-15.

Deaton, A., and J. Muellbauer. "An Almost Ideal Demand System." Amer. Econ. Rev. 70(June 1980):312-26.

De Boer, P. M. C., and R. Harkema. "Some Evidence on the Performance of Size Correction Factors in Testing Consumer Demand Models." Econ. Letters 29(1989)(4):311-15.

Denny, M., and M. Fuss. "The Use of Approximation Analysis to Test for Separability and the Existence of Consistent Aggregates." Amer. Econ. Rev. 67(June 1977):404-18.

Diewert, W. E., and T. J. Wales. "Flexible Functional Forms and Global Curvature Conditions." Econometrica 55(January 1987):43-68.

Eales, J. S., and L. J. Unnevehr. "Demand for Beef and Chicken Products: Separability and Structural Change." Amer. J. Agr. Econ. 70(August 1988):521-32.

Goldman, S., and H. Uzawa. "A Note on Separability in Demand Analysis." Econometrica 32(July 1964):38798.

Gorman, W. M. "Separable Utility and Aggregation." Econometrica 27(July 1959):469-81.

Green, R., and J. M. Alston. "Elasticities in AIDS Models." Amer. J. Agr. Econ. 72(May 1990):442-45.

Gregory, A. W., and M. R. Veall. "Formulating Wald Tests on Nonlinear Restrictions." Econometrica 53(November 1985): 1465-68.

Hayes, D., T. I. Wahl, and G. Williams. "Testing Restrictions on a Model of Japanese Meat Demand." Amer. J. Agr. Econ. 72(August 1990):556-66.

Italianer, A. "A Small-Sample Correction for the Likelihood Ratio Test." Econ. Letters 19(1985)(4):315-17.

Jorgenson, D. W., and L. J. Lau. "The Structure of Consumer Preferences.” Ann. Econ. Soc. Measur. 4(Winter 1975):49-101.

LaFontaine, F., and K. J. White. "Obtaining any Wald Statistics You Want." Econ. Letters 21(1986)(1):35-40.

LaFrance, J. "When is Expenditure 'Exogenous' in Separable Demand Models?” West. J. Agr. Econ. 16(July 1991):49-62.

Laitinen, K. "Why is Demand Homogeneity So Often Rejected?" Econ. Letters 1(1978)(3):187-91.

Leontief, W. "Introduction to a Theory of the Internal Structure of Functional Relationships." Econometrica 15(October 1947):361-73.

Lewbel, A. "AIDS, Translog, and the Gorman Polar Form." Econ. Letters 24(1987)(2):161-63.

Meisner, J. F. "The Sad fate of the Asymptotic Slutsky Symmetry Test for Large Systems." Econ. Letters 2(1979)(3):231-33.

Moschini, G. "A Non-Nested Test of Separability for Flexible Functional Forms." Rev. Econ. Stat. 74 (May 1992):365-69.

Moschini, G., and A. Vissa. "The Linear Inverse Demand 
System.” J. Agr. Res. Econ. 17( December 1992):294302.

Mountain, D. C. "The Rotterdam Model: An Approximation in Variable Space." Econometrica 56(March 1988):477-84

Nicol, C. "The Effect of Expenditure Aggregation on Hypothesis Tests in Consumer Demand Systems." Int Econ. Rev. 32(May 1991):405-16.

Pudney, S. E. "An Empirical Method of Approximating the Separable Structure of Consumer Preferences." Rev. Econ. Stud. 48(October 1981):561-77.

Sono, M. "The Effects of Price Changes on the Demand and Supply of Separable Goods." Int. Econ. Rev. 2(September 1961):239-71.
Theil, H. The Theory and Measurement of Consumer Demand. Volume I. Amsterdam: North-Holland, 1975. . The Theory and Measurement of Consumer Demand. Volume II. Amsterdam: North-Holland, 1976.

- The System-Wide Approach to Microeconomics. Chicago: The University of Chicago Press, 1980.

Uzawa, H. "Production Functions with Constant Elasticity of Substitution." Rev. Econ. Stud. 29(October 1962):291-99.

Varian, H. "Non-parametric Tests of Consumer Behaviour." Rev. Econ. Stud. L(January 1983):99-110.

Winters, L. "Separability and the Specification of Foreign Trade Functions.” J. Int. Econ. 17(November 1984): 239-63. 